\title{
Slave-boson approach to the size-dependent transition from van der Waals to covalent bonding in $\mathrm{Hg}_{n}$ clusters
}

\author{
M.E. Garcia, K.H. Bennemann \\ Institut für Theoretische Physik der Freien Universität Berlin, Amimallee 14, 14195 Berlin, Germany
}

(Received 13 August 1993; accepted 11 January 1994)

\begin{abstract}
We use a microscopic theory to describe the dynamics of the valence electrons in divalent-metal clusters. The theory is based on a many-body model Hamiltonian $H$ which takes into account, on the same electronic level, the van der Waals and the covalent bonding. In order to study the ground-state properties of $H$ we have developed an extended slave-boson method. We have studied the bonding character and the degree of electronic delocalization in $\mathrm{Hg}_{n}$ clusters as a function of cluster size. Results show that, for increasing cluster size, an abrupt change occurs in the bond character from van der Waals to covalent bonding at a critical cluster size $n_{\mathfrak{c}} \sim 10-20$. This change also involves a transition from localized to delocalized valence electrons, as a consequence of the competition between both bonding mechanisms.
\end{abstract}

The size dependence of the electronic structure of $\mathrm{Hg}_{n}$ clusters is one of the most fascinating problems of cluster physics. The mercury atom is characterized by having a closed-shell electronic configuration of the form $[\mathrm{Xe}] 6 \mathrm{~s}^{2}$, i.e., isoelectronic to helium, with a relatively large atomic gap to the empty $6 p$ level. At the other end of the size dependence, mercury bulk shows metallic properties due to the overlap of the $s$ and $p$ bands. Thus, from the point of view of the electronic structure in the atom, small $\mathrm{Hg}_{n}$ clusters should be expected to be van der Waals bonded. However, since the bulk band widths, and consequently the hopping elements, are large, one cannot exclude the possibility of covalent bonding even in the very small clusters. Thus, in order to study the size dependence of the bond character and degree of electronic delocalization in $\mathrm{Hg}_{n}$ clusters, a microscopic theory is needed, which must be able to treat both the covalent and the van der Waals bonding mechanisms on the same electronic level. It is the purpose of this paper to present such a theory.

A correct description of the size evolution of the electronic structure of small $\mathrm{Hg}_{n}$ clusters only in terms of a single-particle theory is not possible. The intuitive (single-particle like) picture of a gradually increasing broadening of the $s$ and $p$ bands (with the consequent reduction of the $s p$ gap) due to an increase of the average coordination number, is in contradiction with experiment [1-5]. The experimental results for size dependence of the $5 d \rightarrow 6 p$ autoionization energy [1] ionization potential $[2,3]$, cohesive energy and optical properties $[4,5]$, show a common feature, which is a relatively abrupt change in the be- 
haviour at a given critical cluster size $n_{c}$. In particular, the cohesive energy of $\mathrm{Hg}_{n}$ clusters [3] can be well fitted by using Lennard-Jones potentials for $n \leq 13$. For $n>13$ the cohesive energy increases more rapidly and the agreement becomes worse as the cluster size increases.

A consistent interpretation of the experiments has been performed [6], with the nontrivial conclusion, that in small $\mathrm{Hg}_{n}$ clusters the valence electrons remain almost completely localized until a critical cluster size $n_{c}\left(10 \leqslant n_{c} \leqslant 20\right)$, where there is a transition to delocalized electronic states. This abrupt transition reflects the fact that small $\mathrm{Hg}_{n}$ clusters are strongly correlated systems.

In order to find which correlations are important for this transition we consider the main electronic interactions present in neutral $\mathrm{Hg}_{n}$ clusters, which are included in the following many-body model Hamiltonian [7]

$H=H_{\text {cov }}+H_{\text {vdl }}+H_{\mathrm{QP}}$.

$\mathrm{H}_{\text {cov }}$ describes the covalent interactions by a generalized sp-band Hubbard Hamiltonian of the form

$$
\begin{aligned}
H_{c o v}= & \sum_{l \beta \sigma \sigma} \epsilon_{\beta} c_{l \beta \sigma}^{+} c_{l \beta \sigma r}+\sum_{l j \beta \gamma \sigma \sigma} t_{\beta \gamma}^{l j} c_{l \beta \sigma r}^{+} c_{j \gamma \sigma} \\
& +\sum_{l \beta \sigma \sigma \neq \gamma \sigma \sigma^{\prime}} U_{\beta \gamma} n_{l \beta \sigma r} n_{l \gamma \sigma^{\prime}},
\end{aligned}
$$

where $\epsilon_{i \beta}\left(\beta=\mathrm{s}, \mathrm{p}_{x}, \mathrm{p}_{y}, \mathrm{p}_{z}\right)$ refers to the atomic-like energy levels, $t_{\beta \gamma}^{l i}$ to the different interatomic hopping integrals, and $U_{\beta \gamma}$ to the on-site direct Coulomb-interaction integrals. $c_{i \beta \sigma}^{+}$, $c_{i \beta_{v}}$ and $n_{l \beta_{r r}}$ are the corresponding creation, annihilation and occupation-number operators. Note that the covalent interactions in neutral $\mathrm{Hg}_{n}$ clusters involve mainly inter-atomic sp hop- (a)

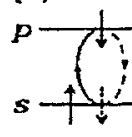

1

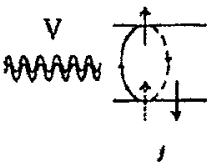

f (b)

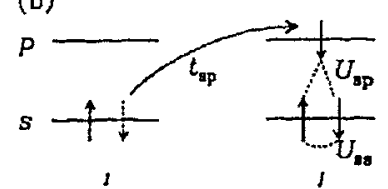

Fig. 1. Illustration of the dominant electronic excitations responsible for (a) van der Waals interactions (involving localized electrons) and (b) covalent interactions (interatomic hopping). ing. In Eq. (1), the operator $H_{\mathrm{vdw}}$ describes the van der Waals interactions as arising from the coupling between intraatomic sp excitations (which is a microscopic description of the induced dipole-dipole interactions), and has the form $[7,8]$ :

$$
\begin{aligned}
H_{\mathrm{vdW}}= & \frac{1}{2} \sum_{l \neq j, p \sigma \sigma^{\prime}} V_{l j}^{(1)} c_{l \mathrm{p} \sigma}^{+} c_{l \mathrm{~s} \sigma} c_{j \mathrm{p} r^{\prime}}^{+} c_{j \mathrm{~s} \sigma^{\prime}} \\
& -\frac{1}{2} \sum_{l \neq j, p \sigma \sigma^{\prime}} V_{l j}^{(2)} c_{l \mathrm{p} \sigma}^{+} c_{l \mathrm{~s} \sigma^{\prime}} c_{j \mathrm{~s} \sigma^{\prime}}^{+} c_{j \mathrm{p} \sigma^{\prime}}+\text { h.c., }
\end{aligned}
$$

where $V_{l j}^{(1)}=V_{l j}^{(2)} \alpha \alpha \Delta / r_{j l}^{3}$, are the coupling constants for the van der Waals interactions $[7,8]$. Here $\alpha$ refers to the atomic polarizability, $\Delta$ to the atomic sp gap and $r_{j l}$ to the interatomic distance. The operator $H_{\mathrm{QP}}$ in Eq. (1) describes the charge-dipole interactions and is given by

$H_{\mathrm{QP}}=\sum_{l, j} Q_{l} \frac{r_{l j} \cdot P_{j}}{r_{l j}^{3}}$

In Eq. (4), $Q_{l}=2-|e| \sum_{\beta \bar{\sigma}} n_{l \beta \sigma}$ refers to the charge operator at atom $l$, and $P_{l}=e \sum_{\beta \gamma c r} X_{\beta \gamma}$ $c_{l \beta e r}^{+} c_{l y, r}$ stands for the dipole operator at atom $l$, where the components of $X_{\beta \gamma}$ are the atomic dipole matrix elements between orbitals $\beta$ and $\gamma$. In Fig. 1, the intraatomic and interatomic electronic processes responsible for the van der Waals and covalent bonding, and represented by the operators $H_{\text {viw }}$ and $H_{\text {cov }}$, respectively, are schematically shown. Clearly, since the same valence electrons take part on both cohesion processes, an interplay between vdW and covalent bonding arises, which dominates the electronic structure and is contained in the Hamiltonian $H$.

The calculation of the ground-state properties of $H$ requires one to take into account explicitly the different local electronic configurations which contribute to the correlated ground-state wave function. Therefore, we introduce, in analogy with the slave-boson method [9] a set of boson operators which project onto the different possible atomic configurations. This means that one needs $16 \times n$ boson operators to describe a $\mathrm{Hg}_{n}$ cluster [8]. However, only a few of these local configurations contribute appreciably to the ground state properties of neutral $\mathrm{Hg}_{n}$ clusters. Therefore, we 
consider the creation (annihilation) operators $s_{\sigma}^{+}$ $\left(s_{v}\right), d_{s}^{+}\left(d_{\mathrm{s}}\right), \tau_{\mathrm{p} \sigma}^{+}\left(\tau_{\mathrm{p} \sigma}\right)$ and $m_{\sigma \bar{\sigma}}^{+}\left(m_{\sigma \bar{\sigma}}\right)$, which correspond, respectively, to the configurations $s^{1}$, $s^{2}, s^{2} p^{1}$ and $s^{1} p^{1}$ (with opposite spins). Of course, in order to obtain a one to one correspondence between bosons and local electronic configurations, a set of constraints has to be imposed to the boson operators [7,8]. Thus, in the enlarged Hilbert space (including fermions and bosons) one obtains a Hamiltonian $\mathscr{H}$ which has the same matrix elements as the original (pure electronic) Hamiltonian $H$. For instance, the transformations [8]

$c_{j \gamma \sigma}^{+} c_{l \beta \sigma} \longrightarrow c_{j \gamma \sigma}^{+} c_{l \beta \sigma} Z_{j \gamma \sigma}^{+} Z_{l \beta \sigma}$,
$c_{l \mathrm{p} \sigma}^{+} c_{l s \sigma} c_{j \mathrm{p} \sigma}^{+} c_{j \mathrm{~s} \sigma} \longrightarrow c_{l \mathrm{p} \sigma}^{+} c_{l \mathrm{~s} \sigma} c_{j \mathrm{p} \sigma}^{+} c_{j \mathrm{~s} \sigma} \Omega_{l \sigma}^{+} \Omega_{j \sigma}^{+}$,

where $Z_{j \gamma \sigma}^{+}, Z_{l \beta \sigma}$ and $\Omega_{l \sigma}^{+}$are operators acting on the boson space, provide the generalizations of the original fermion operators describing interatomic hopping and coupling between two intraatomic sp excitations. The Coulomb energy in the enlarged space can be written only in terms of boson operators, as [8]

$E_{C o u l}=U \sum_{l \sigma} \tau_{l p \sigma}^{+} \tau_{l p \sigma}$

We evaluate the ground state properties in the saddle-point approximation (SPA) [9]. Here, the boson operators are taken to be numbers determined by minimizing the ground-state energy. By setting $s_{\sigma}^{+} s_{v}=s^{2}, \quad d_{s}^{+} d_{s}=d^{2}, \quad \tau_{\mathrm{p} \sigma}^{+} \tau_{\mathrm{p} \sigma}=\tau^{2}$ and $m_{\sigma \bar{\sigma}}^{+} m_{\sigma \bar{\sigma}}=m^{2}$, we recover an electronic effective Hamiltonian $H_{\text {eff }}$, in which the hopping elements and the van der Waals coupling constant become renormalized, by the factors $[7,8]$

$q^{\prime}=\left\langle Z_{l \beta \sigma}^{+} Z_{j \gamma \sigma}\right\rangle$

and

$q^{\nu}=\left\langle\Omega_{l v}^{+} \Omega_{j \sigma^{\prime}}^{+}\right\rangle$

which depend on the numbers $s^{2}, d^{2}, \tau^{2}$ and $m^{2}$, which can be interpreted as the probabilities for the occurrence of the different atomic configurations. This reflects in a very transparent way the interplay between covalent and van der Waals bonding. Moreover, it holds that $0 \leq q^{t}, q^{V} \leq 1$. Thus, one can interpret the renormalization fac- tors as order parameters. The effective Hamiltonian $H_{\text {eff }}$ can be easily diagonalized $[7,8]$ and its ground state energy $E_{n}\left(d^{2}, s^{2}, \tau^{2}, m^{2}, \ldots\right)$ is given by

$$
\begin{aligned}
E_{n}= & 2 \sum_{\beta=\mathrm{s}, \mathrm{p}} \int_{-\infty}^{\mathrm{HOS}} \mathrm{d} \epsilon \tilde{\mathscr{N}}_{\beta}(\epsilon)+2 n U \tau^{2} \\
& +\sum_{l, j} \frac{\left(q^{V} V\right)^{2}}{\tilde{\epsilon}_{s}-\tilde{\epsilon}_{p}} \\
& +2 n \sum_{\mu} \lambda_{\mu} f_{\mu}\left(\tau^{2}, d^{2}, s^{2}, m^{2}\right) .
\end{aligned}
$$

The first term of Eq. (10) is the kinetic energy of the electrons, where $\tilde{\mathscr{N}}_{s(p)}(\epsilon)$ refers to the renormalized $s(\mathrm{p})$ density of states. In the second (Coulomb) term, $U$ is the Coulomb energy of a charge fluctuation, and $\tau^{2}$ the probability for the ionic (triply occupied) configurations. The third term stands for the van der Waals energy, and the last term refers to the constraints $f_{\mu}=0$ or $f_{\mu}=$ fermion operator, which the probabilities $s^{2}$, $d^{2}, \tau^{2}$ and $m^{2}$ have to satisfy. The quantities $\lambda_{\mu}$ are the corresponding Lagrange multipliers. The binding energy of the cluster is then obtained by minimizing the function $E_{n}\left(d^{2}, s^{2}, \tau^{2}, m^{2}, \ldots\right)$ with respect to the probabilities $s^{2}, d^{2}, \tau^{2}$ and $m^{2}$, subjected to the corresponding constraints.

We have performed calculations for $\mathrm{Hg}_{n}$ clusters with $n \leq 43$ atoms and for different values of the parameters $U$ and $V$. Since both $v d W$ and covalent bonding favour close-packed structures, we have assumed such cluster geometries. For $n \geq 13$ results on fcc-like structures are presented in this paper. No qualitative difference have been found for icosahedral structures. In general, results were checked to be insensitive to the details of the cluster structure.

The parameters $\epsilon_{s}, \epsilon_{\mathrm{p}}, t_{\gamma \beta}^{l j}$, used for the calculations are determined as follows. The sp-promotion energy $\Delta=\epsilon_{\mathrm{p}}-\epsilon_{\mathrm{s}}=5.8 \mathrm{eV}$ is obtained from the average between the ${ }^{3} \mathrm{P}$ and ${ }^{1} \mathrm{P}$ atomic $6 \mathrm{~s}^{2} \rightarrow$ $6 \mathrm{~s} 6 \mathrm{p}$ transitions [10]. We use $t_{\mathrm{ss}}=-0.44 \mathrm{eV}, t_{\mathrm{sp}}$ $=0.5 \mathrm{eV}$, and $t_{\mathrm{pp}}=0.73 \mathrm{eV}$, similar to those of Ref. [6], which were fitted to bulk-Hg assuming the relation $t_{\mathrm{ss} \sigma}=-1.32 / 1.42 t_{\mathrm{sp} \sigma}=-1.32 / 2.22$ $t_{\mathrm{pp} \sigma}$ proposed by Harrison [11]. Due to the constraints for the probabilities, $E_{n}$ is actually func- 


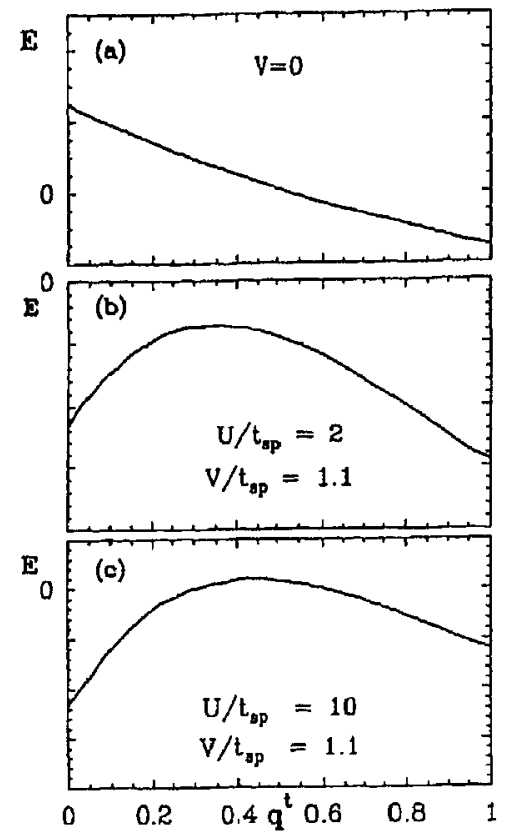

Fig. 2. Schematic plot of the lowest value of the function $E_{n}$ $(n=2)$ for each pair $\left(d^{2}, m^{2}\right)$ as a function of the hopping renormalization factor $q^{t}$ for different values of $U$ and $V$ (scaled by $t_{\mathrm{sp}}$ ). A crossing of the energy minima occurs for increasing $U$.

tion of only two variables, for example $d^{2}$ and $m^{2}$ $[7,8]$ Definite values of the hopping and $\mathrm{vdW}$ coupling renormalization factors $q^{t}$ and $q^{V}$ correspond to each point $\left(d^{2}, m^{2}\right)$.

For $V=0$ we obtain, for all cluster sizes, a single minimum in $E_{n}\left(\mathrm{~m}^{2}, d^{2}\right)$ having $q^{t}=1$ and $q^{V}=0$ (see Fig. 2). The probability for the $s-p$ mixed state in this minimum is negligible $\left(m^{2} \sim 0\right)$. The binding is, thus, exclusively due to interatomic charge fluctuations. For increasing Coulomb repulsion, it results that the probability for the triply (singly) occupied state is $\tau^{2}=s^{2} \sim$ $t_{\mathrm{sp}}^{2} /(\Delta+U)^{2}$ for $U \rightarrow \infty$. This means that the binding energy, which comes from charge fluctuations, decreases for increases $U$. The energy of this saddle point $E_{n}\left(q^{l}=1\right)$ decreases for increasing $U$ as

$E_{n} \sim-\frac{t_{\mathrm{sp}}^{2}}{\Delta+U} \rightarrow 0$ for $U \rightarrow \infty$.

This minimum corresponds, as expected, to the saddle point (SP) solution of a two-band Hubbard model with 2 electrons per site. The behaviour of $E_{n}$ described in Eq. (11) for large $U$ coincides with the binding energy up to second order perturbation theory, i.e., it has the same dependence with $U$ as the exact solution of a Hubbard Hamiltonian. New features appear if vdW interactions are included. For $V \neq 0$, in addition to the covalent minimum, a second $\mathrm{SP}$ is found in $E_{n}$ having $q^{V}=1$ and $q^{t}=0$, i.e., pure vdW character. This is shown schematically for $n=2$ in Fig. 2(b), where we plot the lowest value of $E_{n}\left(d^{2}, m^{2}\right)$ for each value of $q^{t}\left(d^{2}, m^{2}\right)$. In this SP the probability of triple and single occupations is negligible $\left(\tau^{2}=s^{2} \sim 0\right)$, whereas $m^{2} \sim(V / \Delta)^{2} \neq 0$. This indicates that the binding arises from intraatomic electron-hole excitations. The energy of this $y d W$ SP is independent of $U$, and is approximately given by

$E_{n} \sim-V^{2} / \Delta$,

i.e., London vdW cohesion. The SP which is actually relevant for the cluster ground state is the one with the lowest energy. For the parameters used in Fig. 2(b) this is the covalent SP. For the same value of $V$ but increasing $U$, the energy of the covalent SP decreases according to (11), until a value $U_{\mathrm{c}}$ is reached, for which both minima have the same energy. With a further increase of $U$ the vdW SP becomes the absolute minimum, as illustrated in Fig. 2(c). At $U_{c}$ the ground state of the cluster changes dramatically its properties and undergoes a transition from delocalized, covalent $\left(q^{t}=1\right)$ to localized, vdWlike $\left(q^{t}=0, q^{\nu}=1\right)$ electronic states.

It is important to stress, that for $V=0$, i.e., in absence of vdW interactions, the electrons are delocalized $\left(q^{t}=1\right)$ for all cluster sizes $n$. This indicates that the correlations introduced by the van der Waals forces are of fundamental importance and must be considered in order to account for the experimental observations.

It is possible to obtain, for each cluster size, a phase diagram for the bond character as a function of $U$ and $V$, as shown in Fig. 3 for a cluster of $n=13$ atoms. Clearly, there are many ways to go from the van der Waals to the covalent phase. For instance, by keeping $U$ constant and decreasing $V$ until a critical value $V_{c}$ is reached for which the system changes from covalent to vdW bonding. For decreasing $U, V_{\mathrm{c}}$ needed to sup- 


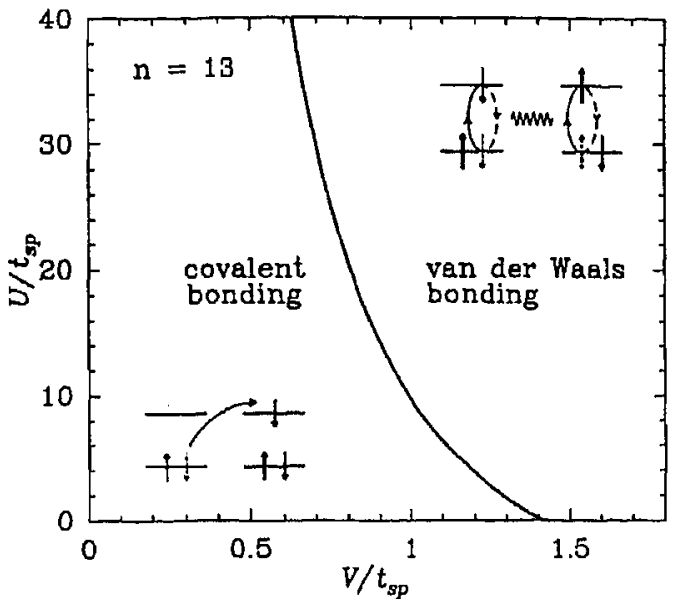

Fig. 3. Phase diagram for $v d W$ and covalent bonding as a function of the Coulomb energy of a charge fluctuation $U$ and the vdW coupling constant $V$ (both scaled by $t_{\mathrm{sp}}$ ) for $n=13$ atoms.

press charge fluctuations increases. This reflects clearly the important role of the Coulomb repulsion as regulating the interplay between covalent and $\mathrm{vdW}$ bonding. Other possible path for such a transition consists in keeping $V$ constant while $U$ is reduced. And this case is particularly important, since there is a very simple way to decrease $U$, namely by increasing the cluster size. One expects $U$ to decrease due to the increase of the screening of the charge fluctuations. In our theory, the screening is given by the charge-dipole interactions $H_{\mathrm{Q}-\mathrm{P}}$ [Eq. (4)]. As shown schematically in Fig. 3, in a hopping process a positive and a negative ions are formed. The Coulomb energy $U$ that the electrons have to pay is given by

$U=U_{0}-e^{2} / r_{i j}-\delta U_{l j}$,

where $U_{0}=2 U_{\mathrm{sp}}-U_{\mathrm{ss}}$ is the increase of the intra-atomic Coulomb repulsion, $-e^{2} / r_{i j}$ the interatomic attraction between the electron and the remaining hole, and $\delta U_{l j}$ takes into account the screening of the interatomic charge fluctuation due to the polarization of the atoms surrounding the electron-hole pair $[7,8]$. This polarization of the environment is clearly a size-dependent effect. Since the polarization energy increases with increasing cluster size [7,8], we conclude that a transition from van der Waals to covalent bonding can occur for increasing cluster size.

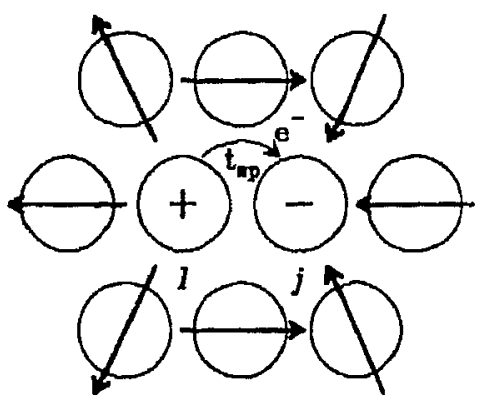

Fig. 4. Screening process consisting in polarization of the neutral atoms surrounding a charge fluctuation. Thick arrows refer to the induce dipole moments.

In order to explain quantitatively the change in the bond character of $\mathrm{Hg}_{n}$ clusters as a function of cluster size, we estimate the interaction parameters $V$ and $U$ as follows. The vdW coupling constant $V=c_{1} \alpha \Delta / r_{0}^{3}=0.55$ is obtained from the atomic polarizability [12] $\alpha=5.7 \AA^{3}$, and the constant $c_{1}=1.25$, which was determined by fitting to the experimental binding energy of rare-gas dimers. For simplicity we assume $U_{\mathrm{ss}}=U_{\mathrm{sp}}=U_{0}$. In Fig. 5 results are given for the bond character of $\mathrm{Hg}_{n}$ clusters as a function of $U_{0}$ and $n$. The phase boundary is determined as the value of $U_{0}$ for which $n$ is equal to the critical cluster size $n_{\mathrm{c}}$, where the transition occurs. A realistic value

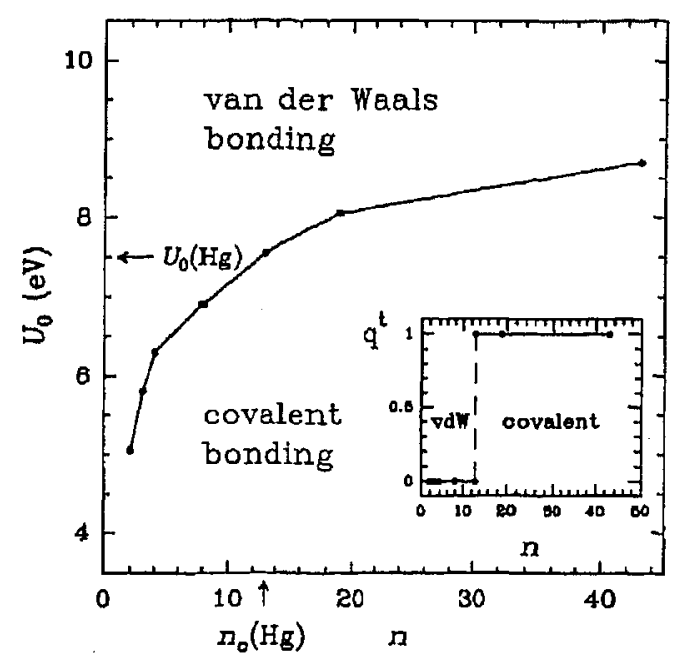

Fig. 5. Phase diagram for vdW and covalent bonding as a function of the intraatomic Coulomb repulsion $U_{0}$ and $n$. Estimating $U_{0}(\mathrm{Hg})=7.5 \mathrm{eV}$, one obtains $n_{\mathrm{c}}(\mathrm{Hg})=13$ atoms. The subfigure shows the size dependence the hopping renormalization $q^{t}$. 
$U_{0}(\mathrm{Hg})=7.5 \mathrm{eV}[10,13]$ is indicated. This yields a transition at $n=13$ atoms. Of course, since we use a model Hamiltonian, the set of parameters used cannot be determined accurately to describe univocally $\mathrm{Hg}$. Therefore, we allow an uncertainty in the value of $U_{0}$ of approximately $1 \mathrm{eV}$. Then, we can conclude that the transition occurs in $\mathrm{Hg}_{n}$ clusters at $n_{c} \approx 10-20$ atoms, in very good agreement with experiment. In the inset of Fig. 5, the size dependence of the degree of delocalization of the valence electrons (renormalization factor $q^{t}$ ) is shown. For $n \leq 13, q^{t}=0$ implies the hopping processes are blocked. The number of single and triple occupations vanishes, i.e., the electrons are localized. In addition, $q^{V}=1$ reflects that only vdW bonding is present. The binding energy and the number of p-electrons per bond is roughly independent of $n$. At $n=13$ there is a crossing of the SP solutions and the character of the bonding changes. For $n>13$ we obtain $q^{t}=1$, which physically means that the valence electrons delocalize to form covalent bonds (or for even larger clusters metallic bonds) and vdW bonding is suppressed $\left(q^{V}=0\right)$. Now, the binding energy per bond increases with $n$, and the same occurs with the number of p-electrons.

The change in the bond character of $\mathrm{Hg}_{n}$ clusters is, of course, also reflected in the size dependence of the cohesive energy, as it has been shown in a previous paper [7], where good quantitative agreement with experiment is obtained.

Although the results for $U_{c}(n)$ depend not only on $n$ and $U_{0}$, but also implicitly on the other parameters $\left(V, \Delta, t_{\mathrm{sp}}\right)$, Fig. 5 can be interpreted as a general phase diagram for the size dependent bond character in divalent-metal clusters. Notice that the existence of a transition is restricted to certain values of $U_{0}$. For instance, if $U_{0}<5 \mathrm{eV}$, the system could be covalent already for $n=2$ [14]. For $U_{0}>9 \mathrm{eV}$, vdW behaviour results for all cluster sizes.

We study now the change of the bonding in rare-gas clusters. From Refs. [10,11,13] and by fitting to experimental results of rare-gas dimers we estimate the values $U_{0}(\mathrm{Xe})=9.13 \mathrm{eV}, \Delta(\mathrm{Xe})$ $=13.26 \mathrm{eV}, \alpha(\mathrm{Xe})=4.04 \AA^{3}$, and $r_{0}(\mathrm{Xe})=4.36$ $\AA$ for $\mathrm{Xe}_{n}$ clusters, $U_{0}(\mathrm{Kr})=10.48 \mathrm{eV}, \Delta(\mathrm{Kr})=$ $17.11 \mathrm{eV}, \alpha(\mathrm{Kr})=2.48 \AA^{3}$, and $r_{0}(\mathrm{Kr})=4.03 \AA$, for $\mathrm{Kr}_{n}$ clusters and $U_{0}(\mathrm{Ar})=11.12 \mathrm{eV}, \Delta(\mathrm{Ar})=$ $18.7 \mathrm{eV}, \alpha(\mathrm{Ar})=1.64 \AA^{3}$, and $r_{0}(\mathrm{Ar})=3.76 \AA$ for $\mathrm{Ar}_{n}$ clusters. We assume that $\mathrm{Xe}_{n}, \mathrm{Kr}_{n}$ and $\mathrm{Ar}_{n}$ clusters have the same hopping elements as $\mathrm{Hg}_{n}$ clusters, which is an overestimation, since the interatomic distances between rare-gas atoms are larger than between $\mathrm{Hg}$ atoms, and since the overlap between atomic-like wave functions is known to be smaller than for $\mathrm{Hg}$ [15]. In spite of it we obtain, in the SPA, that $\mathrm{Xe}_{n}, \mathrm{Ar}_{n}$ and $\mathrm{Kr}_{n}$ clusters are vdW for all cluster sizes investigated. This means that in our model, rare-gas clusters are can be seen as divalent-metal clusters for which the transition to covalent bonding does not take place.

Summarizing, we have explained the size dependent change of the bonding character in small $\mathrm{Hg}_{n}$ clusters by using an electronic theory which takes into account properly the correlations introduced by the interplay between van der Waals and covalent bonding mechanisms.

\section{Acknowledgements}

We would like to thank Dr. G.M. Pastor for his contribution to the development of this theory. An important part of this work was done in collaboration with him. This work has been supported by the Deutsche Forschungsgemeinschaft, through SFB 337.

\section{References}

[1] C. Bréchignac, M. Broyer, Ph. Cahuzac, G. Delacretaz, P. Labastie, J.P. Wolf and L. Wöste, Chem. Phys. Lett. 120 (1985) 559, and Phys. Rev. Lett. 60 (1988) 275.

[2] K. Rademann, B. Kaiser, U. Even and F. Hensel, Phys. Rev. Lett. 59 (1987) 2319.

[3] H. Haberland, H. Kornemeier, H. Langosch, M. Oschwald and G. Tanner, J. Chem. Soc. Faraday Trans. 86 (1990) 2473.

[4] K. Rademann, O. Dimopolou-Rademann, M. Schlauf, U. Even and F. Hensel, Phys. Rev. Lett. 69 (1992) 3208.

[5] H. Haberland, B. von Issendorff, Ji Yufeng and T. Kolar, Phys. Rev. Lett. 69 (1992) 3212.

[6] G.M. Pastor, P. Stampfli and K.H. Bennemann, Physica Scripta 38 (1988) 623; Europhys. Lett. 7 (1988) 419. 
[7] M.E. Garcia, G.M. Pastor and K.H. Bennemann, Phys. Rev. Lett. 67 (1991) 1142.

[8] M.E. Garcia, Ph.D. Thesis, Freie Universität Berlin, 1992.

[9] G. Kotliar and A.E. Ruckenstein, Phys. Rev. Lett. 57 (1986) 1362.

[10] C. Moore, Atomic Energy Levels, Nat. Bur. Stand. Circ. No. 467 Vol. II (1952) and Vol. III (1958).

[11] W.A. Harrison, Phys. Rev. B 24 (1981) 5835.

[12] Handbook of Chemistry and Physics, 66th Edition (CRC, Boca Raton, Florida, 1985).
[13] W.A. Harrison, in Electronic Structure and the Properties of Solids (W.H. Freeman and Company, San Francisco, 1980); Phys. Rev. B 31 (1985) 2121.

[14] This could be the case of $\mathrm{Be}$. Although $U_{0}(\mathrm{Be})>5 \mathrm{eV}$, the atomic gap $\Delta$ in $\mathrm{Be}$ is much smaller than in $\mathrm{Hg}$. Furthermore, $\mathrm{Be}_{n}$ clusters have a shorter interatomic distance $r_{0}$, and consequently large overlap and hopping elements.

[15] R.O. Jones, J. Chem. Phys. 71 (1979) 1300. 